\title{
Protée
}

\section{Présentation : Lumières}

\section{Marie Renoue}

Volume 31, numéro 3, hiver 2003

Lumières

URI : https://id.erudit.org/iderudit/008432ar

DOI : https://doi.org/10.7202/008432ar

Aller au sommaire du numéro

Éditeur(s)

Département des arts et lettres - Université du Québec à Chicoutimi

ISSN

0300-3523 (imprimé)

1708-2307 (numérique)

Découvrir la revue

Citer cet article

Renoue, M. (2003). Présentation : Lumières. Protée, 31(3), 5-6.

https://doi.org/10.7202/008432ar d'utilisation que vous pouvez consulter en ligne.

https://apropos.erudit.org/fr/usagers/politique-dutilisation/ 
PRÉSEN TATIO N

\title{
LUMIÈRES
}

\author{
MARIE RENOUE
}

"I would like to say that there never is no light», dit James Turrell ${ }^{1}$, dont la production artistique est consacrée à la lumière sous ses différentes manifestations physiques et physiologiques. Cette ubiquité, comme également la valeur symbolique de la lumière associée aux notions de clarté intellectuelle, d'évidence, auraient pu être l'indice d'une simplicité du phénomène, de sa description et de ses interprétations. Pourtant, toute impression d'évidence s'évanouit dès que l'on tente de préciser ce qu'est la lumière, ses valeurs sémantiques, mais aussi ce qu'il en est de sa visibilité - contestée entre autres par le philosophe O. Revault d'Allonnes, qui écrit:

La situation est quelque peu paradoxale: si la lumière est la condition indispensable pour qu'un objet soit visible, en revanche la lumière elle-même ne se voit pas. [... L La lumière à la fois existe et $n^{\prime}$ existe pas. ${ }^{2}$

Aussi la lumière fut-elle et est-elle encore le lieu ou l'objet de spéculations philosophiques, cosmologiques, théologiques ou scientifiques - que l'on songe au mythe platonicien de la Caverne, au feu héraclitéen, à la lumière non astrale du Fiat Lux de la Genèse ou au photon, ondulatoire ou corpusculaire, de la physique quantique. Elle est un enjeu axiologique, esthétique, donc éthique, ainsi que le soulignent le fameux Éloge de l'ombre de J. Tanizaki (1933) et nombre d'édifices religieux. Objet sémiotique de choix pour le sémioticien, elle l'oblige, par sa trop grande polyvalence, à préciser un angle d'approche, à circonscrire un champ de manifestation, ainsi que le feront les diverses contributions à ce dossier de Protée, intitulé «Lumières".

Au-delà de la diversité des objets étudiés, le dossier «Lumières » présente des convergences thématiques et une variété méthodologique et théorique: il s'agit d'interroger les régimes de visibilité de la lumière, sa capture comme objet de valeur ou ses fonctions comme opérateur de visibilité d'objets, d'interroger également les valeurs différentielles du plan de l'expression ou du contenu qui la définissent, ses manifestations paradoxales ou complexes et synesthésiques. Il s'agit en fait de traiter de l'émergence du lumineux et de la lumière, de l'émergence d'un objet sémiotique, voire d'une approche sémiotique qui puisse le prendre en charge - car l'un des enjeux de ce dossier est assurément la confrontation de démarches sémiotiques déjà reconnues ou innovantes et en devenir.

Essentiellement consacré à la lumière des arts, ce dossier s'ouvre néanmoins avec une contribution traitant de «lumière naturelle», celle de $P$. Boudon, qui propose d'exfolier des figures de lumière astrale sous la forme diagrammatique des templa, afin d'élaborer une sémiotique de la physique dégagée de 
I'anthropomorphie des approches narratives et passionnelles courantes en sémiotique - et qui seront représentées ci-après.

Les autres contributions offrent l'examen d'objets artistiques singuliers ou pluriels plus ou moins intégré à l'explicitation d'une démarche sémiotique (une sémiotique passionnelle et tensive, une étude aspectuelle et actantielle des régimes de visibilité de la lumière). J. Fontanille propose ainsi I'analyse d'un effet: «la lumière intérieure» des paysages baudelairiens en termes actantiels et passionnels, pour mettre en évidence les deux configurations spatio-temporelles qui participent à la constitution sémiotique du paysage («le paysage-existence» et « le paysage-expérience» indépendant ou dépendant de l’observateur). W. Fiers fait une analyse interactive des contraintes physiques de la lumière - la morphogenèse - et épistémologiques de la perception - la sémiogenèse - en termes de structures sensori-motrices à partir de deux sculptures de verre de Raphaël Farinelli et d'Étienne Leperlier.

Distincte de la narrativisation greimassienne de la signification, la sémiotique tensive que C. Zilberberg propose pour sa part, dont il expose les principes - la référence rhétorique, le primat de l'intensité - avant une analyse de la structure de la luminosité, de «styles» lumineux et « de la productivité de la noirceur», est apparente entre autres dans l'Éloge de l'ombre de J. Tanizaki et dans l'œuvre d'outrenoir de Pierre Soulages. Marie Renoue traite aussi des relations paradoxales entre le noir et la lumière en évoquant les peintures de Pierre Soulages, mais aussi les Lights Pieces de James Turrell, dont la dimension haptique est soulignée. L'haptique et le synesthésique sont les points d'ancrage de l'étude de la texture représentée ou ostensive que réalise A. Beyaert, attentive à son interdéfinition avec les autres dimensions du visible et à son exploitation dans les arts du Xxe siècle. Enfin, F. Parouty-David consacre son étude à la lumière représentée, à ses tensions et à ses valeurs actantielles, synesthésiques et axiologiques, dans La Mort de la Vierge du Caravage, le peintre par excellence de la dialectique ombre et lumière.

Puisque la lumière est également un matériau à travailler ou un objet façonné par des spécialistes de l'éclairage, les témoignages de praticiens ne pouvaient être oubliés. Aussi la section «Documents» propose-t-elle deux textes: une interview réalisée par P.-E. Parais et M. Renoue avec le chef-opérateur de la «nouvelle vague», R. Coutard, qui évoque les contraintes ou recherches techniques qui ont participé à la formation des lumières ayant fait sa renommée; et le texte de J.-J. Ezrati, qui distingue pratiques et métiers de la lumière, avant de présenter une de ses réalisations muséales de concepteur éclairagiste.

Une pratique de la lumière dont V. Laganier, architecte, éclairagiste et photographe, donne des exemples dans le dossier iconographique qu'il a réalisé ici.

La responsable du dossier remercie chaleureusement tous les auteurs, Raoul Coutard pour son aimable disponibilité et Vincent Laganier pour sa participation esthétique.

1. E. Laaksonen: «James Turrell art minimal and conceptual only- interview with James Turrell », 1996: http://www.home.sprynet.com/ mindweb/page44.htm. 2. «Les obscures évidences de la lumière», Revue d'esthétique, nº 37 ("De la lumière»), 2000, p. 9. 